\title{
Perspective
}

PERSPECTIVE Actualité en histoire de l'art

$2 \mid 2015$

Les États-Unis

\section{Au-delà de la visualité : retours critiques sur la matérialité et l'affect}

\section{Veerle Thielemans}

Traducteur : Françoise Jaouën

\section{OpenEdition \\ Journals}

\section{Édition électronique}

URL : http://journals.openedition.org/perspective/6171

DOI : $10.4000 /$ perspective.6171

ISSN : 2269-7721

\section{Éditeur}

Institut national d'histoire de l'art

\section{Édition imprimée}

Date de publication : 31 décembre 2015

Pagination : 141-147

ISBN : 978-2-917902-27-1

ISSN : $1777-7852$

\section{Référence électronique}

Veerle Thielemans, «Au-delà de la visualité : retours critiques sur la matérialité et l'affect », Perspective [En ligne], 2 | 2015, mis en ligne le 30 juin 2017, consulté le 01 octobre 2020. URL : http:// journals.openedition.org/perspective/6171; DOI : https://doi.org/10.4000/perspective.6171 


\section{Au-delà de la visualité : retours critiques sur la matérialité et l'affect}

\section{Veerle Thielemans}

Telle qu'elle est pratiquée aux États-Unis, l'histoire de l'art est une discipline largement autoréflexive qui privilégie l'établissement de généalogies, la clarification méthodologique et le positionnement critique. Les chercheurs se sentent souvent contraints de se joindre à des débats autour de sujets jugés pertinents pour l'époque, et de prendre clairement position. Au cours des dernières décennies, l'un des débats les plus récurrents a porté sur le rôle joué par le visuel. Depuis les années 1970, un grand nombre d'études très diverses ont été consacrées à l'évolution des conditions historiques du regard et à la dimension idéologique de la représentation visuelle. Le public français connaît surtout les noms de T. J. Clark, Jonathan Crary, Michael Fried, Rosalind Krauss et W. J. T. Mitchell, mais on pourrait citer bien d'autres chercheurs ${ }^{1}$. Aujourd'hui, l'investigation semble s'orienter vers une nouvelle direction. On délaisse la dimension visuelle de l'œuvre pour s'intéresser de manière croissante à son aspect proprement matériel et à sa réception sensorielle et affective ; celle-ci retrouve ainsi son statut d' " objet ", un objet composé de matériaux divers, que l'on déplace, que l'on installe à tel ou tel endroit, que l'on retire, que l'on échange, etc. Parallèlement, un nouveau vocabulaire expressif se crée afin de décrire l'expérience de regarder une œuvre dans ses dimensions multi-sensorielle et affective. Le retentissement émotionnel de cette expérience fait désormais partie intégrante du processus interprétatif, et celui qui regarde est un spectateur en chair et en os et non plus un " œil désincarné ".
Ce délaissement de la visualité a été en quelque sorte préparé de l'intérieur par le biais d'une critique de la neutralité des opérations du regard et de la représentation visuelle. Les études postmodernes des années 1980 et 1990 s'en prenaient au privilège accordé au regard depuis l'avènement de la modernité, considérant que le paradigme visuel des sociétés occidentales répondait à des codes historiques et sociaux. En montrant que l'accent mis sur la dimension optique pure permettait d'exercer une forme de contrôle sur la subjectivité individuelle, ces études dévoilaient le soubassement idéologique de ce régime visuel, remettant ainsi en question la transparence supposée de celui-ci. Dans son étude sur les techniques disciplinaires organisant la vision dans la culture européenne au début du XIX ${ }^{\mathrm{e}}$ siècle, Crary a été l'un des premiers à démontrer que l'accent mis sur les modes d'observation visuelle témoignait d'un malaise créé par les nouvelles

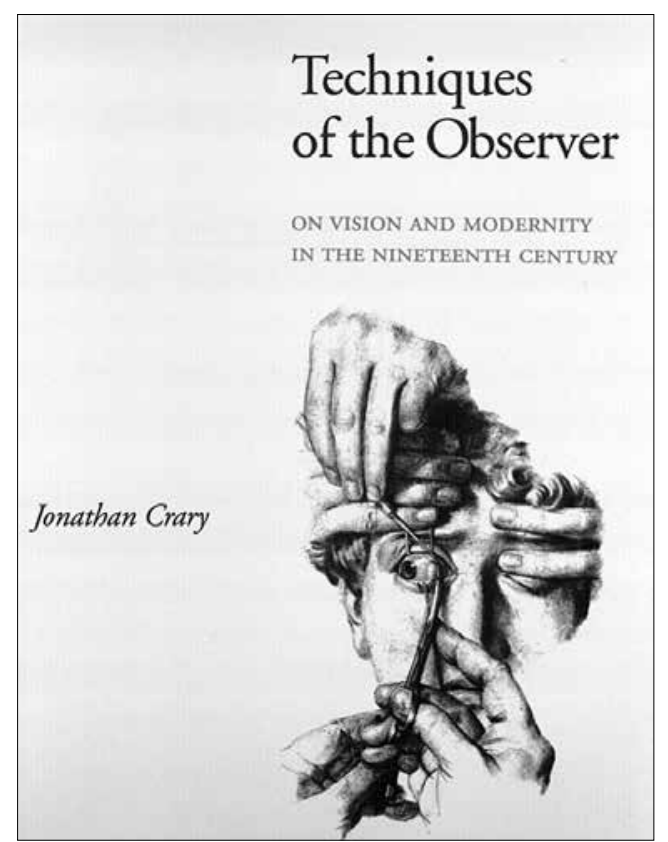

1. Jonathan Crary, Techniques of the Observer: On Vision and Modernity in the Nineteenth Century, Boston, 1990. 
2. W. J. T. Mitchell, Que veulent les images? Une critique de la culture visuelle, Dijon, 2014 p. $320-321$.

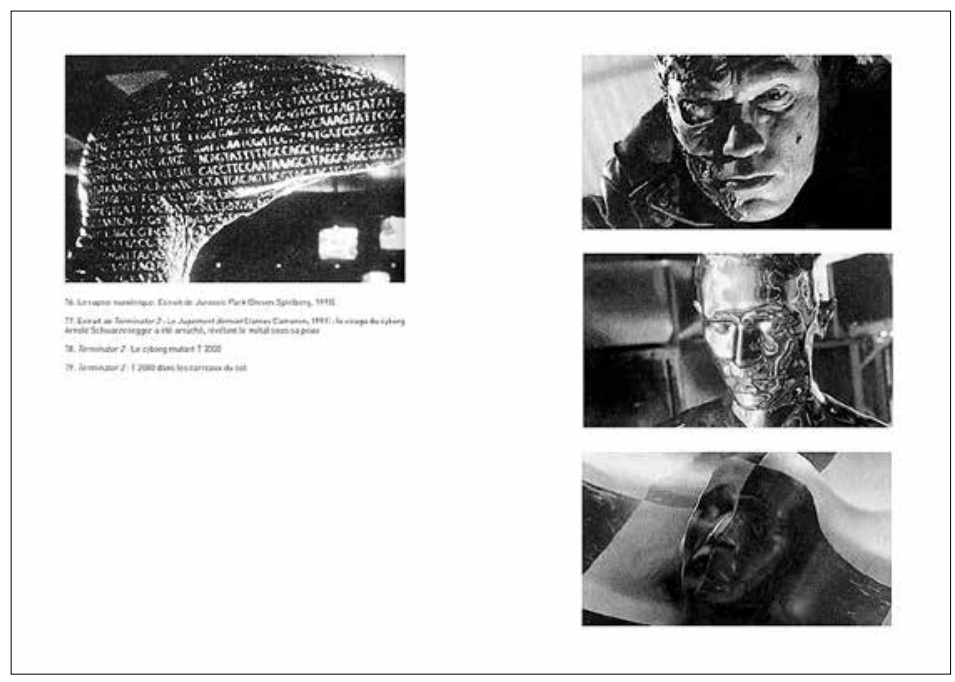

les conventions représentationnelles, le dialogue que les œuvres entretiennent avec l'art du passé, etc. Je songe ici aux travaux de Mieke Bal (Double Exposures: The Subject of Cultural Analysis), de Barbara Stafford (Good Looking: Essays on the Virtue of Images), de James Elkins (The Object Stares Back) et de Michael Ann Holly (Past Looking: Historical Imagination and the Rhetoric of Images) $)^{5}$. Dans les études visuelles, cette prise en compte croissante de la complexité du travail

découvertes dévoilant l'aspect incontrôlable des mécanismes physiologiques chez l'homme (y compris de l'esprit inconscient; fig. 1) ${ }^{2}$. Dans la même veine, les recherches sur les questions de race et de genre, ainsi que le féminisme et les études queer ont porté un oil critique sur l'aspect normatif des approches culturelles du corps, et dénoncé le déséquilibre des relations de pouvoir qui en découle ${ }^{3}$. En outre, les recherches sur les répercussions de l'expérience du deuil et des violences physiques et psychiques, qui ont débuté en histoire de l'art dans les années 1990 dans le cadre des trauma studies, ont largement contribué à mettre en évidence la pression exercée par les événements douloureux, dont l'empreinte non visuelle influe tant sur la création de l'œuvre que sur le regard porté sur celle-ci ${ }^{4}$.

De surcroît, dans la nouvelle discipline constituée par les études visuelles, on a introduit de subtiles distinctions qui font de la visualité un paradigme nettement moins envahissant. En 1996, notamment, la revue October a publié un "Visual Culture Questionnaire " dans lequel les répondants émettent de nombreuses réserves vis-à-vis du privilège accordé à l'œil dans les analyses pratiquées. La même année, plusieurs ouvrages majeurs ont plaidé pour une approche plus complexe du travail de l'image. Ils mettaient en avant l'effet produit par les conditions physiques du regard - où interviennent le désir, des moments d'aveuglement, le surgissement de souvenirs enfouis - ainsi que le rôle joué par la manière d'exposer l'objet artistique, des images s'observe tout particulièrement chez W. J. T. Mitchell. Après avoir étudié la manière dont la théorie a fait de l'image une affaire de nature ou de convention, et plaidé pour l'analyse des mécanismes idéologiques qui les transforment en signes naturalisés, il s'est progressivement intéressé à la fascination qu'elles exercent ${ }^{6}$. Dans Que veulent les images?, il démontre avec brio que les images sont perçues comme étant simultanément mortes et vivantes, qu'il s'agisse des idoles les plus primitives des sociétés pré-industrielles ou des produits technologiques complexes d'aujourd'hui (fig. 2). Objets de vénération et de crainte, elles doivent être considérées comme de véritables agents dans les processus culturels, politiques et économiques en raison de la dimension quasi magique qu'on leur attribue.

L'impulsion théorique qui vise à réorienter les recherches américaines vers la matérialité et l'affect est également venue d'autres disciplines, notamment de l'anthropologie sociale, de l'histoire culturelle et de la philosophie, toutes marquées par la volonté de repenser la position centrale octroyée au langage au sein des humanities $^{7}$ au cours des dernières décennies, et de tester $d^{\prime}$ autres outils conceptuels pour en finir avec ce privilège. La signification, à laquelle aucune limite n'est assignée, devient ainsi en perpétuel devenir. Les relations humaines et non humaines se déploient dans des directions multiples, de manière fluide et non hiérarchique, et fluctuent selon les circonstances et au fil du temps. En référence aux théories anthropologiques de la 
vie sociale des marchandises (Arjun Appadurai) et de " l'agentivité distribuée " (Alfred Gell), les relations sujet/objet deviennent des connexions interactives répondant à des besoins qui s'inscrivent dans un réseau d'échanges matériels et sociaux ${ }^{8}$. La recherche tend désormais à renverser la conception traditionnelle de la relation causale entre activité humaine et " choses " inanimées en dotant d'agentivité les objets eux-mêmes. On s'intéresse désormais à ce qu'ils "déclenchent " et non plus seulement à ce qu'ils " signifient " ${ }^{9}$. L'effet émotionnel des réflexes du système nerveux autonome, et le lien entre ces sensations pré verbales d'une part et les processus de pensée et les actions qui s'ensuivent d'autre part (mécanismes étudiés en psychologie et en neurosciences) sont des notions qui entrent aujourd'hui dans les humanities par le biais de la théorie de $\mathrm{l}^{\prime}$ affect $^{10}$. L'une des sources les plus importantes de la réflexion sur le rôle de l'affect en esthétique est le modèle philosophique de l'expression proposé par Gilles Deleuze et Felix Guattari, selon lesquels la signification surgit d'intenses sensations corporelles qui se cristallisent en unités signifiantes, mais de manière éphémère ${ }^{11}$.

Le champ artistique constitue indéniablement un fertile terrain d'investigation en la matière ; en effet, contrairement à d'autres objets, la " chose " artistique sert de véhicule à la réponse affective, ouvrant ainsi de nouvelles perspectives critiques. Bien entendu, le défi majeur à relever consiste à faire de ces concepts des outils adaptés à l'interprétation historique. Qu'advient-il, par exemple, du critère de la "distance objective " sur lequel se fonde notre discipline lorsque l'on octroie à l'expérience sensible de l'œuvre une place aussi importante dans l'analyse historique ? Quel type de vocabulaire et de structures rhétoriques doiton inventer afin de mettre en mots la perception incarnée (embodied) ? Qu'en est-il de l'intention artistique ou des particularités d'un projet, même si l'on reconnaît que l'artiste n'est plus la figure maîtresse du triangle "producteur/œuvre/ spectateur " ? La déconstruction de la figure de l'auteur par le structuralisme et le poststructuralisme a entraîné une critique des processus et des systèmes qui attribuent une signification aux choses. Ce type d'investigation a-t-il encore sa place lorsqu'on s'éloigne des questions sociales et politiques, ou doit-on s'en tenir à sa propre réponse subjective à l'œuvre ?
L'attention portée à la matérialité de l'œuvre d'art et de l'effet que celle-ci produit sur le spectateur constitue certes un rééquilibrage bienvenu, car l'histoire sociale de l'art, qui domine la discipline depuis de longues années aux États-Unis, repose, pour une très large part, sur le matériel contextuel, parfois au détriment de la composition matérielle de l'œuvre. Mais ce nouvel intérêt ne signifie pas pour autant que l'on doive revenir à l'analyse formelle, contrairement à ce que semblent craindre les critiques du " tournant matériel "; il peut se combiner à l'investigation de la signification sociale de l'œuvre, de la visée artistique, etc. Dans le domaine des American studies, des travaux ambitieux ont ainsi été menés entre les années 1970 et 1990 par des chercheurs formés à Yale University - lieu important de croisement entre l'histoire de l'art et l'étude matérielle de la culture - notamment autour de la figure de Jules Prown ${ }^{12}$. Quant aux travaux très personnels d'Alexander Nemerov sur Raphaelle et Rembrandt Peale, Timothy O'Sullivan, ou encore Edward Hopper, ils tracent des liens inattendus entre biographie individuelle, histoire collective et expression artistique ${ }^{13}$. Les écrits de Nemerov se distinguent tout autant par leur qualité littéraire et l'attention portée au détail que par les configurations historiques innovantes qu'ils exposent. On pourrait également citer le nom de Jennifer Roberts, qui a travaillé sur la peinture et l'illustration de la fin du XVIII ${ }^{e}$ siècle

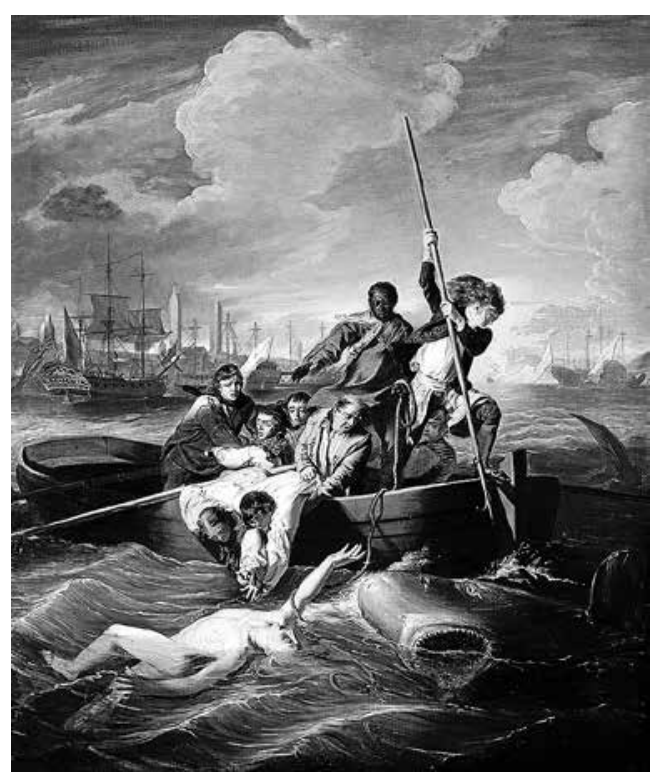

3. John Singleton Copley, Watson and the Shark, 1778, Washington, D.C., National Gallery of Art. 


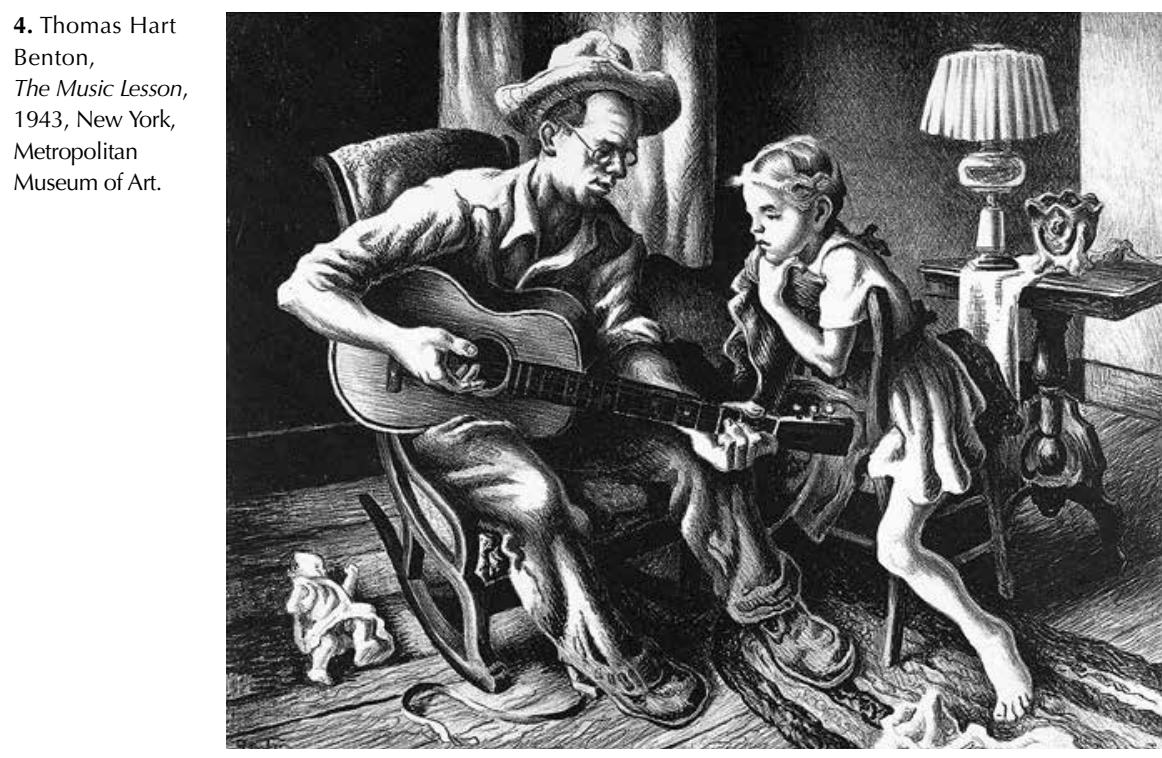

et du début du XIX ${ }^{e}$ siècle $^{14}$. Elle voit dans les conditions matérielles du " transit " de ces objets une forme d' « agentivité " (qui s'exprime notamment dans l'œuvre de John Singleton Copley de façon métaphorique ; fig. 3), rapprochant ainsi l'histoire des arts américains de l'époque des préoccupations actuelles sur les échanges commerciaux, les technologies de reproduction et les systèmes de communication. Elle ouvre ainsi de nouvelles perspectives sur l'histoire traditionnelle de la naissance d'une nation et d'un art, un récit longtemps resté figé dans l'histoire de l'art des débuts de l'Amérique.

S'inspirant des études sur l'histoire culturelle des sens relevant de l'anthropologie, d'intéressantes recherches ont également été menées sur l'expression/la représentation de l'expérience sensorielle autre que visuelle. Caroline A. Jones fait ici figure de pionnière avec sa critique incisive du primat accordé à l'œil propre à la critique issue du haut modernisme, et l'intérêt qu'elle porte aux expérimentations artistiques qui élargissent le spectre sensoriel, notamment le body art et la performance $^{15}$. La réception sensorielle est également devenue un champ d'investigation pour les chercheurs qui s'intéressent à l'impact des nouveaux médias - le sentiment, par exemple, d'être plongé dans une émission radiophonique ou une conversation téléphonique, deux phénomènes devenus des motifs de la peinture américaine des premières décennies du XX siècle (fig. 4$)^{16}$. Ce cadre de réflexion ouvre la voie à de nouvelles interprétations en mettant en relief des récits devenus trop familiers, ou en soulignant certains éléments jusquelà délaissés. On songe notamment aux multiples recherches menées sur les années 1960 et 1970, recherches où le modèle de la perception incarnée a donné lieu à de fructueuses relectures de la performance, de l'installation et du cinéma expérimental, ainsi que de formes artistiques où l'expérience sensorielle semble jouer un rôle mineur (l'art conceptuel, par exemple $)^{17}$. La théorie de l'affect a attiré l'attention d'un nombre croissant d'historiens de l'art américains qui ont poussé plus loin les recherches sur le corps et l'expérience incarnée. Jusqu'ici, les explorations les plus fécondes semblent concerner les études sur le cinéma, la danse et la performance, où la communication non verbale s'effectue par le biais du corps en mouvement ${ }^{18}$.

En s'alignant sur les théories centrées sur l'objet - anthropologie, culture matérielle, études sur les médias et philosophie - l'histoire de l'art américaine est peut-être en train d'évoluer de manière décisive. Il est encore trop tôt pour savoir sur quoi déboucheront ces investigations, mais on repère d'ores et déjà les principaux domaines où les changements sont les plus notables. Au cours des dernières décennies, les recherches américaines dans les domaines de l'histoire sociale de l'art, des études sur l'identité et le genre, des études féministes et des queer studies, ainsi que de l'histoire des institutions et des études visuelles ont pour une large part privilégié l'analyse de l'ordre social afin d'étudier le lien entre l'art et son public ; aujourd'hui, le concept d' " agentivité " occupe une place de plus en plus importante et fournit une nouvelle donne. En décembre 2009, à l'occasion d'une conférence qu'il donnait dans le cadre du programme sur l'histoire sociale de l'art à l'Insitut national d'histoire de l'art, j'avais demandé à T. J. Clark si les œuvres d'art pouvaient être dotées d'agentivité. " Non », m'avait-il clairement 
répondu avec un sourire amusé. Aujourd'hui, nombre d'historiens seraient d'un avis contraire. L'idée que les images/objets sont dotés d'agentivité a introduit une nouvelle dimension temporelle dans les travaux qui s'intéressent à la capacité mnémonique de l'art. Si les recherches portent encore largement sur la reconstitution du contexte historique, on reconnaît cependant que les objets artistiques ont le pouvoir de faire resurgir du passé des couches signifiantes enfouies grâce à l'impact affectif produit sur celui qui les contemple ${ }^{19}$. La recherche américaine se rapproche ainsi de la tradition française et allemande en histoire de l'art, tradition qui s'appuie sur les modèles du temps historiques définis par Aby Warburg et Walter Benjamin, selon lesquelles le lien entre passé et présent est de nature " anachroniste " et échappe à la chronologie classique ${ }^{20}$. Il n'est pas inutile de souligner ce point car faire de $\mathrm{l}^{\prime}$ « anachronisme » un concept-clé dans l'analyse de la contemplation d'une ouvre en tant que rencontre sensitive peut sembler paradoxal dès lors que l'expérience temporelle est mise en relief. Michael Ann Holly, ancienne directrice du Clark Research Institute et farouche défenseur des visual studies américaines, fait une éloquente démonstration de ce qui se joue dans la rencontre affective avec l'art du passé. Dans ses travaux précédents, elle traçait des parallèles entre l'échafaudage rhétorique du texte de l'historien de l'art et la structure formelle de l'œuvre; dans son dernier livre, The Melancholy Art, elle montre que c'est le passé lui-même qui façonne le texte (fig. 5) ${ }^{21}$, et le travail de l'historien est de libérer cette force et de la rendre intelligible. Ce processus est guidé par un sentiment de perte, affirme-t-elle ; l'écriture de l'histoire de l'art se transforme en quête des origines, en recherche de ce qui n'est plus ${ }^{22}$. Enfin, en faisant de l'expérience une condition du travail interprétatif, la subjectivité - si longtemps bannie de l'investigation savante - trouve une nouvelle place. Il s'agit sans doute là d'un des aspects les plus étonnants (et potentiellement contestables) des évolutions à venir. Il resterait encore beaucoup à dire sur les raisons pour lesquelles la subjectivité redevient désirable ; on pourrait aussi se demander en quoi elle se démarque du rôle traditionnel assigné à l'auteur, et comment la discipline, ses méthodes et ses objectifs en sont affectés. Il serait notamment utile de savoir si cette subjectivité diffère des avatars

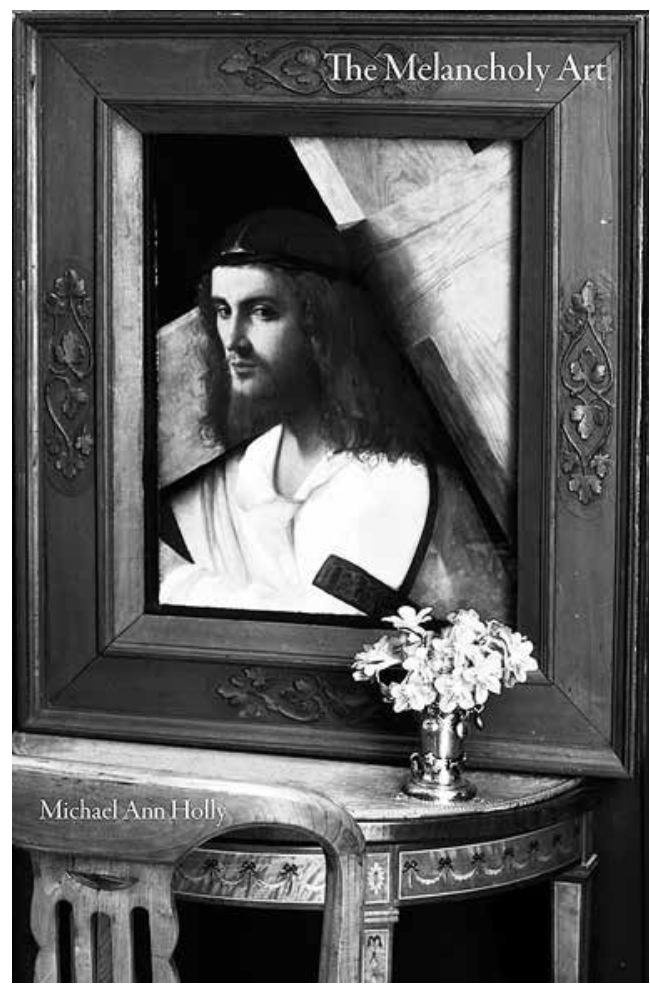

5. Michael Ann Holly, The Melancholy Art, Princeton, 2013.

précédant la critique poststructuraliste, et dans quelle mesure. Les prémisses se sont modifiées. Nous avons davantage conscience de la position relative que nous occupons dans le monde. Nous reconnaissons l'opacité du langage et des choses et le caractère médiat des relations sociales. En faisant du chercheur une caisse de résonance ou un capteur sensible, on pourrait peut-être rapprocher l'histoire de l'art américaine et l'art lui-même, en se gardant d'oublier les apports de la critique sociale. Un pas qui mériterait sans doute d'être franchi.

Cet article a été traduit par Françoise Jaouën. 
Je suis reconnaissante à Elisabeth Glassman, Darby English et Amy Zinck, qui m'ont aidé à trouver le temps et l'espace nécessaires à ce projet.

1. Parmi les textes de référence, citons notamment T. J. Clark, The Painting of Modern Life: Paris in the Art of Manet and his Followers, Princeton, 1985 ; Jonathan Crary, L'Art de l'observateur : vision et modernité au XIX siècle, Nîmes, 1994 [éd. orig. : Techniques of the Observer: On Vision and Modernity in the Nineteenth Century, Boston, 1990] ; James Elkins, The Domain of Images, Ithaca, 1999 ; David Freedberg, Le Pouvoir des images, trad. Alix Girod, G. Montfort, 1998 [éd. orig. : The Power of Images: Studies in the History and Theory of Response, Chicago, 1989] ; Michael Fried, Contre la théâtralité : $d u$ minimalisme à la photographie contemporaine, Paris, 2007 [éd. orig. : Art and Objecthood: Essays and Reviews, Chicago, 1998] ; Rosalind Krauss, L'Inconscient optique, Paris, 2002 [éd. orig. : The Optical Unconscious, Cambridge, 1994] ; W. J. T. Mitchell, Iconologie : image, texte, idéologie, Paris, 2009 [éd. orig. : Iconology: Image, Text, Ideology, Chicago, 1986] ; W. J. T. Mitchell, Picture Theory: Essays on Verbal and Visual Representation, Chicago, 1994 ; W. J. T. Mitchell, Que veulent les images? Une critique de la culture visuelle, Dijon, 2014 [éd. orig. : What do Pictures Want? The Lives and Loves of Images, Chicago, 2005] ; Michael Podro, Depiction, New Haven/Londres, 1998 ; Hal Foster éd., Vision and Visuality, Seattle, 1988 ; Norman Bryson, Michael Ann Holly, Keith Moxey éd., Visual Theory: Painting and Interpretation, New York, 1991.

\section{Crary, (1990) 1994, cité n. 1.}

3. Parmi de très nombreux ouvrages, citons notamment : Eve K. Sedgwick, Épistémologie du placard, Paris, 2008 [éd. orig. : Epistemology of the Closet, Berkeley, 1990] ; Douglas Crimp, Melancholia and Moralism: Essays on AIDS and Queer Politics, Cambridge, 2002 ; Jennifer Doyle, Sex Objects: Art and the Dialectics of Desire, Minneapolis, 2006. Bernadette Wegenstein synthétise de manière claire les nouvelles théories sur la corporalité dans "Body ", dans W. J. T. Mitchell, Mark B. N. Hansen éd., Critical Terms for Media Studies, Chicago, 2010, p. 19-34.

4. Voir, entre autres, Hal Foster, Le Retour du réel : la situation actuelle de l'avant-garde, Bruxelles, 2005 [éd. orig. : The Return of the Real: The Avant-garde at the End of the Century, Cambridge, 1996].

5. "Visual Culture Questionnaire ", October, 77, été 1996, p. 25-70 ; James Elkins, The Object Stares Back: On the Nature of Seeing, New York, 1996 ; Mieke Bal, Double Exposures: The Practice of Cultural Analysis, Florence (KY), 1996 ; Michael Ann Holly, Past Looking: Historical Imagination and the Rhetoric of Images, Ithaca, 1996 ; Barbara Stafford, Good Looking: Essays on the Virtue of Images, Cambridge, 1996.

6. Voir les ouvrages cités n. 1.

7. Le terme humanities, souvent traduit par « sciences humaines ", n'a aucun équivalent en français, car s'il englobe notamment les lettres et l'histoire, il exclut en revanche les " sciences " telles que la psychologie et la sociologie (N.D.L.T.).
8. Arjun Appadurai, "Introduction ", The Social Life of Things: Commodities in Cultural Perspective, New York, 1986, p. 3-62 ; Alfred Gell, L'Art et ses agents : une théorie anthropologique, Dijon, 2009 [éd. orig : Art and Agency: An Anthropological Theory, Oxford, 1998]. Pour ces deux auteurs, les systèmes de connaissance et les relations sociales sont davantage déterminés par la circulation des biens que par la production. La "théorie de l'acteur-réseau " de Bruno Latour est également une source importante de cette approche sociale de l'activité humaine.

9. C'est Bill Brown, spécialiste de littérature à University of Chicago, qui a formulé le premier la thing theory (théorie de la chose), qui s'est rapidement propagée à d'autres disciplines des humanities. Voir le numéro spécial de Critical Inquiry consacré à la thing theory, 28/1, automne 2001, et Jane Bennett, Vibrant Matter: A Political Ecology of Things, Durham, 2010.

10. "What If? The Language of Affect ", dans Gilliam Beer, Malcolm Bowie, Beate Perrey éd., In(ter)discipline: New Languages for Criticism, Londres, 2007, p. 6-24; Teresa Brennan, The Transmission of Affect, Ithaca, 2004 ; Melissa Gregg, Gregory J. Seigworth éd., The Affect Theory Reader, Durham, 2010

11. Voir Brian Massumi, "The Autonomy of Affect", dans Paul Patton éd., Deleuze: A Critical Reader, Oxford, 1996, p. 217-39; Paul Patton, Parables for the Virtual: Movement, Affect, Sensation, Durham, 2002 ; Erin Manning, Brian Massumi, Thought in the Act: Passages in the Ecology of Experience, Minneapolis, 2014. Sur le concept d'affect dans les neurosciences, voir Ruth Leys, "The Turn to Affect: A Critique ", dans Critical Inquiry, 37/3, printemps 2011, p. 434-72.

12. Voir Jennifer A. Greenhill, "Response: Look Away ", dans John Davis, J.A. Greenhill, Jason D. LaFountain éd., A Companion to American Art, (Blackwell Companions to Art History, 6), Oxford, 2015, p. 128-45 ; Michael Yonan, "Toward a Fusion of Art History and Material Culture Studies ", dans West $86^{\text {th }}$ : A Journal of Decorative Arts, Design History and Material Culture, 20 septembre 2011 , www.west86th.bgc.bard.edu (consulté le 26 août 2015).

13. Voir les contributions d'Alexander Nemerov, The Body of Raphaelle Peale: Still Life and Selfhood, 1812-1824, Berkeley, 2001 ; " Ground Swell: Edward Hopper in 1939 ", dans American Art, 22, automne 2008, p. 50-71; Acting in the Night: Macbeth and the Places of the Civil War, Berkeley, 2010.

14. Jennifer Roberts, Transporting Visions: The Movement of Images in Early America, Berkeley/Los Angeles, 2014.

15. Caroline A. Jones, Eyesight Alone: Clement Greenberg's Modernism and the Bureaucratization of the Senses, Chicago, 2005 ; Sensorium: Embodied Experience, Technology and Contemporary Art, Caroline A. Jones éd., (cat. expo., Cambridge, MIT Visual Arts Center, 2006-2007), Cambridge, 2006.

16. Voir Leo Mazow, Thomas Hart Benton and the American Sound, University Park, 2012 ; Rachel DeLue, Arthur Dove: Always Connect, Chicago, 2015.

17. Concernant l'art haptique, ce sont les études cinématographiques qui semblent avoir pris les devants; voir Laura U. Marks, The Skin of the Film: Intercultural Cinema, 
Embodiment, and the Senses, Durham, 2000, et Jennifer Baker, The Tactile Eye: Touch and the Cinematic Experience, Berkeley/Los Angeles, 2009. Sur les performance studies, voir, entre autres, l'anthologie dirigée par Sally Banes et André Lepecki, The Senses in Performance, New York/ Londres, 2007. L'historien des médias Fred Turner a analysé la création d'environnements visuels et sonores plaçant de manière prolongée le sujet dans un cadre offrant un choix prédéterminé d'expériences multi-sensorielles en réponse aux évolutions socio-politiques de l'ère de l'information qui visaient à transformer un public réceptif en citoyens démocrates (The Democratic Surround: Multimedia and American Liberalism from World War II to the Psychedelic Sixties, Chicago, 2013). Sur l'art conceptuel, voir Eve Meltzer, Systems We Have Loved: Conceptual Art, Affect, and the Antihumanist Turn, Chicago, 2013 ; l'auteur y affirme que l'art conceptuel peut être envisagé comme une réaction à la disparition de l'humanisme, qui atteint son seuil critique dans les années 1960.

18. Eve K. Sedgwick est parmi les premiers auteurs à avoir introduit les théories de l'affect dans les études féministes et queer ; voir Touching Feeling: Affect, Pedagogy, Performativity, Durham, 2003. Sur la danse et la performance, voir Carrie Noland, Agency and Embodiment: Performing Gestures/Producing Culture, Boston, 2009.

19. Keith Moxey fournit un excellent aperçu des théories récentes sur la manière dont les objets artistiques sont dotés d'agentivité grâce à leurs liens avec le passé ; voir Visual Time: The Image in History, Durham, 2013.

20. L'un des meilleurs ouvrages sur la question est le livre d'Alexander Nagel et Christopher S. Wood, Renaissance anachroniste, Dijon, 2015 [éd. orig. : Anachronic Renaissance, New York, 2010]. Rappelons également que Thomas Crow, spécialiste de l'histoire sociale de l'art, jugeait qu'un "renouveau Warburg " pourrait ouvrir de nouvelles perspectives ("The Practice of Art History in America ", dans Daedalus, 135/2, 2006, p. 87).

21. Holly, 1996; cité n. 5 ; Michael Ann Holly, The Melancholy Art, Princeton, 2013. Une première version du texte est parue en mars 2009 dans The Art Bulletin (89/1), un numéro qui comprenait également d'importants articles sur les conditions de l'écriture historique signés de Stephen Bann, Karen Lang et Hayden White.

22. On retrouve cette tonalité mélancolique chez Nemerov, qui considère que l'histoire de l'art a pour mission ultime de rappeler un monde perdu, de capter les fragments de son apparition et de les retenir grâce à l'écriture : " Être un historien à la manière de la gouvernante, $c^{\prime}$ est tourner, pivoter, montrer du doigt, comme elle, pour diriger l'attention de l'entourage vers un point au sommet de la tour ou sur la grève, là où quelque chose d'effroyable et de dévastateur se dresse 'comme un immense brasier', bien en évidence, et d'entendre les autres demander "Où ça ? ", et redemander sans cesse, car ils ne distingueront jamais rien ("Being a historian in the governess' sense is a matter of turning, swiveling, pointing, just like her, to direct the attention of those around you to a spot atop the tower or on the shore where something devastating and terrible stands 'as big as a blazing fire' as plain as day, only to have those others ask, and never stop asking because they never will see, 'Where?' ", " Seeing Ghosts, 'The Turn of the Screw' and Art History ", dans Maria del Pilar Blanco, Esther Pereen éd., The Spectralities Reader: Ghosts and Haunting in Contemporary Cultural Theory, Londres, 2013, p. 544).

Veerle Thielemans, Terra Foundation for American Art thielemans@terraamericanart.eu 